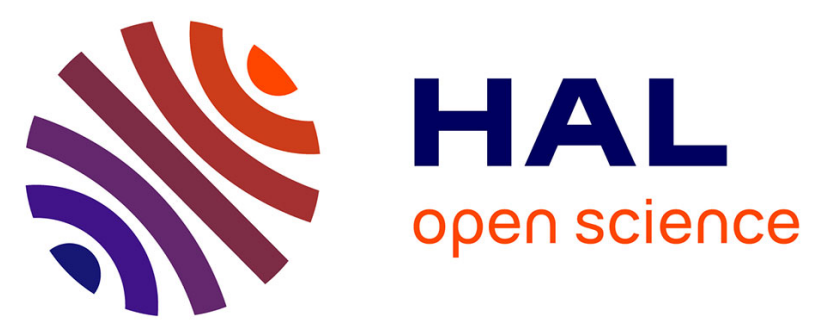

\title{
FOLKBIOLOGY DOESN'T COME FROM FOLKPSYCHOLOGY:EVIDENCE FROM YUKATEK MAYA IN CROSS-CULTURAL PERSPECTIVE
}

Scott Atran, Douglas Medin, Elizabeth Lynch, Valentina Vapnarsky, Edilberto Ucan Ek', Paolo Sousa

\section{- To cite this version:}

Scott Atran, Douglas Medin, Elizabeth Lynch, Valentina Vapnarsky, Edilberto Ucan Ek', et al.. FOLKBIOLOGY DOESN'T COME FROM FOLKPSYCHOLOGY:EVIDENCE FROM YUKATEK MAYA IN CROSS-CULTURAL PERSPECTIVE. Journal of Cognition and Culture, 2001, 1 (1), pp.3-42. ijn_00000128

\section{HAL Id: ijn_00000128 \\ https://hal.science/ijn_00000128}

Submitted on 4 Sep 2002

HAL is a multi-disciplinary open access archive for the deposit and dissemination of scientific research documents, whether they are published or not. The documents may come from teaching and research institutions in France or abroad, or from public or private research centers.
L'archive ouverte pluridisciplinaire HAL, est destinée au dépôt et à la diffusion de documents scientifiques de niveau recherche, publiés ou non, émanant des établissements d'enseignement et de recherche français ou étrangers, des laboratoires publics ou privés. 
FOLKBIOLOGY DOESN'T COME FROM FOLKPSYCHOLOGY:

\title{
EVIDENCE FROM YUKATEK MAYA IN CROSS-CULTURAL PERSPECTIVE
}

\author{
Scott Atran (CNRS, University of Michigan, University of London-Goldsmiths) \\ Douglas Medin and Elizabeth Lynch (Northwestern University) \\ Valentina Vapnarsky (CNRS) \\ Edilberto Ucan Ek’ (Herbolaria Maya Yucatán) \\ Paulo Sousa (University of Michigan)
}




\begin{abstract}
Nearly all psychological research on basic cognitive processes of category formation and reasoning uses sample populations associated with large research institutions in technologically-advanced societies. Lopsided attention to a select participant pool risks biasing interpretation, no matter how large the sample or how statistically reliable the results. The experiments in this article address this limitation. Earlier research with urban-USA children suggests that biological concepts are (1) thoroughly enmeshed with their notions of naive psychology, and (2) strikingly human-centered. Thus, if children are to develop a causally appropriate model of biology, in which humans are seen as simply one animal among many, they must undergo fundamental conceptual change. Such change supposedly occurs between 7 and 10 years of age, when the human-centered view is discarded. The experiments reported here with Yukatek Maya speakers challenge the empirical generality and theoretical importance of these claims. Part 1 shows that young Maya children do not anthropocentrically interpret the biological world. The anthropocentric bias of American children appears to owe to a lack of cultural familiarity with non-human biological kinds, not to initial causal understanding of folkbiology as such. Part 2 shows that by age of 4-5 (the earliest age tested in this regard) Yukatek Maya children employ a concept of innate species potential or underlying essence much as urban American children seem to, namely, as an inferential framework for understanding the affiliation of an organism to a biological species, and for projecting known and unknown biological properties to organisms in the face of uncertainty. Together, these experiments indicate that folkpsychology cannot be the initial source of folkbiology. They also underscore the possibility of a species-wide and domainspecific basis for acquiring knowledge about the living world that is constrained and modified but not caused or created by prior non-biological thinking and subsequent cultural
\end{abstract}


experience. 


\section{Introduction}

The experiments in this article test two competing accounts of how human beings acquire basic knowledge of the everyday biological world, including the categorical limits of the biological domain and the causal nature of its fundamental constituents. ${ }^{1}$ One influential view of conceptual development in folkbiology has been articulated by Susan Carey and her collaborators (Carey 1985, 1995; Carey \& Spelke 1994, Solomon et al. 1996, Johnson \& Solomon 1997, Johnson \& Carey 1998; see also Au \& Romo 1999). On this view, young children's understanding of living things is embedded in a folkpsychological, rather than folkbiological, explanatory framework. Only by age 7 do children begin to elaborate a specifically biological framework of the living world, and only by age 10 does an autonomous theory of biological causality emerge that is not based on children's understanding of how humans think and behave. ${ }^{2}$ A competing view is that folkbiology and folkpsychology emerge early in childhood as largely independent domains of cognition that are clearly evident by ages 4 or 5, and which may be innately differentiated (on folkbiology see Atran 1987, Keil 1989, Gelman \& Wellman 1991, Hatano \& Inagaki 1994; on folkpsychology see Wimmer \& Perner 1983, Leslie 1994, Baron-Cohen 1995, Barrett et al. in press). ${ }^{3}$

Carey's framework combines both similarity-based and causally-based views of folkbiology. In support, she presents evidence for a large shift in patterns of projection for unfamiliar properties over animal kinds. Carey interprets these results as confirming a comparison-to-exemplar model of biological reasoning, wherein humans are the central exemplar or prototype (Carey 1985:28). Such non-normative reasoning patterns are compelling evidence for a particular conceptual framework (Gopnik \& Wellman 1994): specifically, an anthropocentric explanatory framework in which children's conceptions of 
biology are embedded. Children reason about biological phenomena by analogy to humans because they construe the world of living things in a way that is fundamentally different from how adults construe that world. Radical conceptual reorganization is then needed to acquire the purported American adult conceptual system in which humans are "one animal among many." The implications for cognitive psychology are compelling: If children's thought undergoes fundamental conceptual change - a change akin to that seen in scientific revolutions - then it is important to understand the mechanisms underlying this change. These findings also have direct implications for education, because an effective curriculum must take into account the naive, and radically different, theories that young children bring to the classroom.

Despite the importance of this research enterprise for theory and practice, it suffers from a serious limitation. The research has been conducted almost exclusively with majorityculture individuals from North American, urban, technologically-advanced populations. It is important to test the generality of the empirical basis for this model. For example, a knowledge component could well be driving Carey's pattern of results. Her participants preschoolers from the Boston area - probably have little first-hand experience with the natural world. Thus, their human-centered reasoning patterns might reflect lack of knowledge about non-human living things rather than a radically different construal of the biological world. Thus, it is not clear which aspects of children's naïve biology are likely universal and which depend critically on cultural conceptions and conditions of learning. We are also left with little insight into how to best design science curricula for non-majority, non-urban children.

The competing paradigm of an autonomous folkbiology relies to a greater extent on cross-cultural research (Atran 1990; Lopez et al. 1997; see also Berlin et al. 1973; Hunn 
1977; Hays 1983, Brown 1984, Berlin 1992). There is evidence for universal folkbiological principles that are transtheoretical, transcultural, domain-specific, and complex in design. In particular, it appears that people in all societies parse local biodiversity into taxonomies whose fundamental level is that of the "generic species," that is, the level of oak and robin. ${ }^{4}$ In no other natural domain of human understanding does there appear such a unique and fundamental partitioning of reality into mutually exclusive groups that are further organized into mutually exclusive groups within groups (Darwin 1883:363).

For example, in a series of experiments, Coley, Medin and Atran (1997) showed that the taxonomic rank of generic-species is cognitively preferred for biological induction in two diverse populations: people raised in the Midwestern USA and Itza' Maya of the Lowland Mesoamerican rainforest. Their findings cannot be explained by domain-general models of similarity because such models cannot account for why both cultures prefer species-like groups in making inferences about the biological world, although Americans have relatively little actual knowledge or experience at this level. In fact, general relations of perceptual similarity and expectations derived from experience produce a "basic level" of recognition and recall for many Americans that corresponds to the superordinate life-form level of folkbiological taxonomy - the level of tree and bird (Rosch et al. 1976). Still Americans prefer generic species for making inductions about the distribution of biological properties among organisms, and for predicting the nature of the biological world in the face of uncertainty. Additional experiments support the idea of the generic-species level as a partitioning of the ontological domains of plant and animal into mutually exclusive essences that are assumed (but not necessarily known) to have unique underlying causal natures (Atran et al. 1997). ${ }^{5}$

Nevertheless, within this paradigm there has been little direct and controlled study of 
folkbiological acquisition (Dougherty 1979). The few studies that reach beyond the usual sample of children in schools associated with large urban research institutions in the USA (e.g., Hatano \& Inagaki 1999 on Japan) are nonetheless focused on children from urban, technologically-advanced societies. A single study of plant names elicited from Highland Maya children during a nature walk tends to confirm an early childhood preference for identification of generic species; however, the study provides no information about cognitive processes of biological categorization and reasoning or causal structure (Stross 1973).

The experiments reported here represent an initial attempt to address the dearth in cross-cultural studies of folkbiological acquisition. Previous experiments on folkbiological acquisition have often ignored adults (Carey's studies are an exception). Instead, they rely on the implicit familiarity of the experimenter and audience with adults in the standard urbanUSA cultural setting. Without a detailed description of the mature state, however, it is difficult to obtain an accurate picture of the learning curve.

We focus on children and adults of the Yukatek Maya community in southcentral Quintana Roo, Mexico. These are rural folk living in small, lowland villages that have a somewhat dispersed settlement pattern. The area was only fully pacified by the Mexican government at the beginning of the twentieth century, after a rebellion that lasted for more than half a century ("The Caste Wars"). For all participants, Yukatek Maya is the mother tongue. Except for younger children and older women, most participants also speak functional Spanish. Most of the men and older boys are engaged in traditional swidden agriculture, although they also work the surrounding forests both for subsistence and sale of timber and non-timber products. The expansion of the tourist industry from northeast Quintana Roo (e.g., Cancun) into the south has recently begun to siphon off workers from the villages. By and large, women and older girls are engaged exclusively with household 
activities. After the age of six-months, children begin to be initiated into strongly-typed gender roles.

\section{Part I. Induction Studies}

Carey reports three major findings to bolster the claim that children's conceptions of the biological world are anthropocentric. First and foremost, projections from humans are stronger overall than projections from other living kinds. The other two findings are a natural consequence of this difference in induction potential. The second result is that there are asymmetries in projection: inferences from human to mammals are stronger than from mammals to humans. Third, children violate projections according to similarity: inferences from humans to bugs are stronger than from bee to bugs. Together, these findings suggest that humans are the preferred base for children's inferences about the biological world.

In a recent study, Coley, Medin \& James (1999) examined biological induction among rural Native American children (Menominee Indians). The task projection task was patterned after Carey (1985). Children were told that one of the base elements (human, wolf, bee, goldenrod, water) has a certain property inside and then asked whether each of the target elements share this property with the base. The target elements included human, rock and two examples each of mammals (bear, raccoon), birds (eagle, bluejay), reptiles (painted turtle, garter snake), fish (sturgeon, rainbow trout) bug (housefly, earthworm), plant (milkweed, maple) and artifacts (pencil, bicycle).

For young Menominee children humans are a good base and a good target but no more so than are other animals. Coley et al. find no evidence that projections from humans are reliably higher than projections from other bases. Neither do they find significant violations of similarity: inferences from humans to mammals or humans to bugs are no stronger than from mammals to other mammals or bees to bugs. Menominee children see 
humans as one animal among many and not distinct from them (Menominee have an animalbased clan system). In short, it appears that the induction task is sensitive to general knowledge or familiarity with livings kinds as well as specific beliefs about the relation of humans to the rest of nature.

In light of the Menominee results, one possible account of Carey's findings for Boston-area children is that humans are the closest things to non-human animals that urban children know much about and so they generalize from them. ${ }^{6}$ In cultures where children are more familiar with non-human species, there may be little, if any, evidence of an anthropocentric pattern of biological inference at any age. This is the hypothesis that the following experiment was designed to test.

\section{Method}

\section{$\underline{\text { Participants }}$}

Participants were 98 Yukatek Maya-speaking children and 24 Yukatek Mayaspeaking adults from rural villages in southcentral Quintana Roo, Mexico. Forty-six 4-to-5 year olds and forty-eight 6-to-7 year olds were tested. Equal numbers of males and females were included in each group. By and large, the 4-5 year-olds were monolingual, the 6-7 yearolds had begun learning Spanish, and almost all of the adults understood Spanish as a second language. All testing was done in Yukatek Maya.

\section{$\underline{\text { Materials }}$}

Detailed color drawings of objects were used to represent base and target categories. Four bases were used: Human, Dog, Peccary and Bee. Targets were divided into two sets. Each set included a representative of the categories Human (man, woman), Mammal (coatimundi, deer), Bird (eagle, chachalaca), Reptile (boa, turtle), Invertebrate (worm, fly), tree (Kanan, Gumbo Limbo), Stuff (stone, mud), Artifact (bicycle, pencil) and Sun (included 
in both sets). The children were tested on each set at different times, with both sets divided equally among girls and boys. Originally a single mammal base was included, but a second mammal base was included at a later stage when it became apparent that from other ongoing studies that familiarity or domesticity might be a factor. This resulted in some different children being used for the different mammal bases. We included Sun as a target because of its special role in Maya cosmology and society (formerly dominated by "The Sun Priest" = Aj K'in), and because in an earlier pilot study young children overwhelmingly considered the sun to gaze upon people and be "alive."

\section{$\underline{\text { Procedure }}$}

The procedure was similar to that used in Coley et al. Children were shown a picture of one of the bases and taught a new property about it. For example, the experimenter might show the dog picture, and say, "Now, there's this stuff called andro. Andro is found inside some things. One thing that has andro inside is dogs. Now, I'm going to show you some pictures of other things, and I want you to tell me if you think they have andro inside like dogs do." Participants were then shown each of the targets and asked: "Does it have andro inside it, like the [base]?" Properties were unfamiliar internal substances of the form "has X inside." A different property was used for each base, and bases and targets were presented in random order for each participant.

\section{Results}

Each time a child or adult projected a property from a base to a target it was scored 1 , otherwise 0 . Table 1 displays means for targets by base, age group and gender. Responses did not differ reliably across any target pair (e.g., coatimundi vs. deer) and data are collapsed within target types.

Adult Inferences. First, consider the adult data overall. Inferences from Human to other living 
kinds decrease systematically as one moves from mammal to tree. The same systematic change as a function of similarity is also evident for the other two mammal bases (dog and peccary). With bee as a base, inference is strong to other invertebrates, to trees and to humans. Bees often build their nests in trees and humans actively seek out these nests for the honey. The pattern of induction from bee suggest that adults use causal/ecological information in induction, as has been noted before for both Maya and other kinds of biological experts (Lopez et al. 1997, Medin et al. 1997, Atran. 1999; Proffitt, Coley \& Medin 2000).

It is also of interest to compare Human as a base for induction with Dog or Peccary as a base. Adults are more likely to project properties from Dog and Peccary to other mammals than they are to project from Human to other mammals. The picture with respect to asymmetries is somewhat mixed. Inferences going from Human to mammals $(0.65)$ are slightly weaker than inferences from Peccary to humans (0.73) but stronger than inferences from Dog to humans (0.48). A closer look reveals that this weak inference from Dog to humans is largely driven by women ( 0.25 for women versus 0.71 for men). The justifications for dog versus peccary as a base suggests that men consider dogs primarily in terms of forest ecology as a hunting companion and as an animal that hunts, whereas women do not.

One interesting development shift involves an apparent weakening of the category "animal" for adults as a basis for induction. For example, collapsing across age groups, induction from Bee to all other animals (mammals, birds, reptiles and insects) is stronger that induction from Bee to non-animals (tree, stuff, artifact, sun). An ANOVA reveals this main effect: $\mathrm{F}(1,94)=40.35, \mathrm{p}<.001$; however, there is also a AGE GROUP $\mathrm{x}$ TARGET interaction: $\mathrm{F}(2,94)=8.18, \mathrm{p}=.001$. To tease apart the interaction, we performed t-tests that showed the main effect holding for younger children $(\mathrm{t}(25)=2.42, \mathrm{p}=.02)$ and older children 
$(\mathrm{t}(46)=7.65, \mathrm{p}<.001)$, but not adults $(\mathrm{t}(23)=1.7$, n.s. $)$. Adults often cite ecological relationships between bees and the trees they inhabit as a basis for induction.

Children's inferences. Overall, the child and adult induction data are quite similar. Referring again to Table 1, note that for Human, Dog and Peccary as bases, inductive confidence drops as the target moves from mammals to trees. This pattern holds for both groups of children and for all three of these bases. The pattern is weakest for 4-5 year-olds with Human as a base; in this case, the trend does not reach statistical reliability. The fact that the youngest children show a sharp gradient for Dog and Peccary but not Human suggests that the 4-5 year olds do not have a clear conception of where humans fit with the rest of things.

For the older children, humans are inferior to both Dog and Peccary as a base for an induction to mammals. The fact that Dog is a better base for induction than is Peccary suggests that familiarity plays a role in inductive confidence. With respect to asymmetries, the youngest children show a human-mammal asymmetry compared with peccaries $(0.60$ versus 0.29$)$ but not compared with dogs $(0.60$ versus 0.59$)$. In the case of the peccary this asymmetry is mainly driven by the girls who are very unfamiliar with peccaries. Older children show no human to mammals asymmetries.are treated as distinct in that inferences from Dog and Peccary to other mammals are stronger than inferences from Dog and Peccary to humans. This is also true for the adult data.

Older children differ from younger children in showing sharper generalization gradients from mammals as bases. The youngest boys show sharper gradients for Peccary than do the youngest girls. There are no differences in slope when Dog is the base.

Interestingly, girls show a much broader generalization gradient for Human as a base than do boys. This may owe to girls' focus on learning about objects in terms of their household 
roles.

Both ages of children generalize from Bee to other invertebrates, consistent with the use of biological similarity to guide inferences. Surprisingly the youngest girls were much more likely to generalize from Bee to invertebrates than the youngest boys. We have no explanation for this effect.

The category "animal" strongly constrains induction form both younger and older children; however, the sun somewhat weakens this categorical effect for younger children. For example, although t-tests show that projections from Dog and Bee to other animals are significantly stronger than from Dog and Bee to non-animals whether or not the sun is included as a target, projections to non-animals including the sun are also weaker overall than projection to non-animals excluding the sun. Moreover, whereas projection from Peccary to other animals is stronger than projection from Peccary to non-animals excluding the sun $(\mathrm{t}(25)=2.3, \mathrm{p}=.03)$, the difference fails to reach significance if the sun is included among the non-animals $(\mathrm{t}(25)=1.64$, n.s. $)$.

Statistical tests support the above general observations. We group the test by question.

1. Human versus animals as an inductive base to near targets.

a. DOG: Human to mammal (Figure 1) vs. Dog to mammal (Figure 2)

Analysis involved a between subjects ANOVA that included AGE GROUP, GENDER, and ITEM (H->m or D->m). The was only a main effect of ITEM $[F(1,161)=39.75, p=.000]$ and no effects of AGE GROUP or GENDER. The main effect of item is that inferences from Dog to mammal (0.95 across groups) are higher than inferences from Human to mammal ( 0.57 across groups). This is the case for both genders in all groups. These results are inconsistent with Carey's predictions because for all ages inferences from 
Dog to mammal are better than inferences from Human to mammal (compare Figures 1 and 2 to Figures 4 and 5).

b. PECCARY: Human to mammal (Figure 1) vs. Peccary to mammal (Figure 2)

Difference scores were calculated using (Human to mammal) minus (Peccary to mammal). There was only a marginal effect of AGE GROUP $[F(2,91)=2.42, p=.095]$ and no effect of GENDER. Mean difference scores were: Younger children, -0.02 (t-test on $\mathrm{H}->\mathrm{m}$ vs. P->m: $\mathrm{t}(25)=0.20, \mathrm{p}=.85)$; Older children, $-0.30(\mathrm{t}(46)=3.5, \mathrm{p}=.001)$; Adult, -0.17 $(\mathrm{t}(23)=2.0, \mathrm{p}=.06)$. Younger girls on the whole have positive difference scores $(\mathrm{M}=0.13)$, whereas younger boys have negative difference scores $(M=-0.14)$, although the gap between boys and girls does not reach significance.

Positive scores are consistent with violations of similarity. As there were no reliably positive scores, there was no reliable violation of similarity. Nevertheless, there is a marginal developmental shift in which the youngest children are as willing to project from Human to mammals as they are from Peccary to mammals, whereas the older children and adults are more willing to project from Peccary to mammals than from Human to mammals. This shift may, again, have its source in the relative unfamiliarity of the youngest girls with peccaries.

c. BEE: Human to invertebrate (Figure 1) vs. Bee to invertebrate (Figure 3)

Difference scores were calculated using (Bee to invertebrate) minus (Human to invertebrate). With GENDER and AGE GROUP included as factors, again only AGE GROUP was significant $[F(2,91)=3.36, p=.039]$. Mean difference scores were: Younger children, 0.10 (t-test on B->i vs. $\mathrm{H}->\mathrm{i}: \mathrm{t}(25)=.78, \mathrm{p}=.45)$; Older children, $0.32(\mathrm{t}(46)=3.94$, $\mathrm{p}=.000)$; Adults, $0.50(\mathrm{t}(23)=6.28, \mathrm{p}=.0001)$. Posthoc tests show that older children do differ from younger children or adults, but younger children differ from adults.

In this case, negative scores are consistent with Carey. As there are no reliably 
negative scores, there was no reliable violation of similarity (compare Figures 1 and 3 to Figures 4 and 6). Nevertheless, there is again a marginal a developmental shift in which the youngest children are almost as willing to project from Human to invertebrates as they are from Bee to invertebrates, while the older children and adults are more willing to project from Bee to invertebrates than from Human to invertebrates. Bear in mind, though, that the youngest children generalize very broadly and indiscriminately from humans, a finding consistent with the idea that the children do not have a clear conception of how humans fit into the overall folk-taxonomic system.

2. Human versus other animals as an inductive base overall.

To examine whether Human was a preferred inductive base overall we analyzed projections in two different ways. First, we counted the number of targets that each participant projected a base property onto. A BASE (4) by GENDER (2) by AGE GROUP (3) ANOVA revealed only a main effect of BASE: $F(3,43)=16.2, p<.001$. Projection from Human is least common across age groups, and inferences from Dog most common. Post hoc Tukey HSD tests indicate that inference from Human $(M=4.76)$, from Peccary $(M=5.32)$ and from Bee $(M=6.09)$ is each significantly less than inference from $\operatorname{Dog}(M=7.01)$; also inference from Human is reliably less than inference from Bee. Second, we analyzed the target means as function of BASE (4), GENDER (2) and AGE GROUP (3). The ANOVA showed main effects for AGE GROUP, $\mathrm{F}(2,192)=3.813, \mathrm{p}=<.05$, and GENDER, $\mathrm{F}(1,192)$ $=6.095, \mathrm{p}<.05$. Post hoc tests indicate that younger children project properties more readily than adults (older children are not different form younger children or adults). Neither analysis shows inferences from Human to be stronger than those from any other base overall.

Another perspective on reasoning patterns is provided by analyses of how differentiated patterns of generalization are for different bases. We conducted trend analyses to look at 
projection as a function of similarity, with birds were at distance 1 from mammals, reptiles at distance 2 , insects at distance 3 and trees at distance 4 . The factors in the trend analysis were AGE GROUP and SIMILARITY (distance) and our interest was in the linear component.

With Human as a base, there was a main effect of similarity $[\mathrm{F}(1,94)=11.68, \mathrm{p}<$ $0.001]$ and a reliable interaction of age with similarity $[\mathrm{F}(2,94), \mathrm{p}<0.01]$. The interaction arises from the fact that adults show a sharper generalization gradient than either group of children. With Dog as a base there is also a main effect of similarity $[\mathrm{F}(1,73)=241.2, \mathrm{p}<$ $0.001]$ and a group by similarity interaction $[\mathrm{F}(2,73)=4.85, \mathrm{p}<0.05]$. The sharpness of the gradient increases with age but all three groups show reliable similarity effects. For Peccary as a base there is a reliable effect of similarity $[\mathrm{F}(1,94)=39.9, \mathrm{p}<.001]$ but no significant interaction of group with similarity. Finally with Bee as a base there is an effect of similarity $[\mathrm{F}(1,71)=20.14, \mathrm{p}<0.001]$ but again no reliable interaction.

To summarize the trend analyses, all age groups show clear similarity effects with Dog, Peccary and Bee as bases. The generalization (similarity) gradients become sharper with age for Dog as a base. With Human as a base, 4-5 year olds generalize broadly in an undifferentiated manner - they show no reliable effect of similarity. In contrast, adults show characteristically sharp gradients with Human as a base. The 6-7 year olds show a very weak similarity gradient. In short, the clearest developmental change is in determining the role of humans in the folktaxonomic system.

3. Asymmetries in projections to and from humans.

Asymmetry in inference patterns from humans to non-humans versus non-humans to humans is another indication of anthropocentric reasoning. To test this, we analyzed projection from four bases: Human, Dog, Peccary and Bee. Analyses involved ANOVAs and t-tests on difference scores. The dependent variable for each subject was their base to target 
(e.g., Human to mammal) score minus their target to base (e.g., Mammal to human) score.

a. Human-Dog asymmetries. These analyses were between subjects analyses which included AGE GROUP, GENDER, and ITEM ( $\mathrm{H}->\mathrm{m}$ or D $->\mathrm{h})$. This analysis resulted in a AGE GROUP by GENDER interaction $[\mathrm{F}(2,161)=5.38, \mathrm{p}=.005]$ and an ITEM by AGE GROUP by GENDER interaction $[\mathrm{F}(2,161)=5.38, \mathrm{p}=.005]$. To interpret the interaction with gender we repeated this ANOVA separately for males and females.

For males there was no effect of ITEM indicating that there is no asymmetry. There was only an effect of AGE GROUP $[\mathrm{F}(2,79)=3.56, \mathrm{p}=.033]$. The means from Table 1 are: young children $=0.55$, older children $=0.42$, adults $=0.71$. Tukey HSD posthoc tests showed a marginal difference between old children and adults $(\mathrm{p}=.07)$. This means simply that adults overall tended to say "yes" more often than the older children, regardless of item. For females, there was only an ITEM by AGE GROUP interaction $[F(2,82)=4.43, p=.015]$ the means for which are in Table 1. For the youngest girls there is no asymmetry, $\mathrm{t}(24)=.097$, $\mathrm{p}=.654$. For older girls, Dog to human inferences $(\mathrm{M}=0.83)$ are preferred over Human to mammal inferences $(M=0.46), t(36)=2.24, p=.031$. For adults, Human to mammal inferences $(M=0.58)$ are preferred over Dog to human inferences $(M=0.25), t(22)=2.35$, $\mathrm{p}=.028$.

Summary: For males there are no asymmetries: Dog to human inferences and Human to mammal inferences are equally good. For older girls there is a shift with age, but in the direction opposite to that predicted by anthropocentrism. The asymmetry shown by older girls may result from the greater familiarity they have with dogs than with wild mammals (coatimundi, deer) used as the targets in the task (and in the Human to mammal inferences). Because they are familiar with dogs they may be comfortable reasoning about them, whereas their lack of familiarity with the targets makes them less likely to infer things of them. For 
adult women, this familiarity effect may not play a role. These data are inconsistent with Carey because only adult women show the asymmetry favoring Human to mammal over Dog to human (compare Figures 1 and 2 to Figures 4 and 5).

b. Human-Peccary asymmetries. This analysis was done the same way as the previous analysis, with difference scores calculated as: (Human to mammal) minus (Peccary to human). There was an effect of AGE GROUP $[\mathrm{F}(2,91)=3.25, \mathrm{p}=.043]$ and a GENDER $\mathrm{x}$ AGE GROUP interaction $[\mathrm{F}(2,91)=4.92, \mathrm{p}=.009]$. Mean difference scores for were: Younger children, 0.29 (t-test on $\mathrm{H}->\mathrm{m}$ vs. $\mathrm{P}->\mathrm{h}: \mathrm{t}(25)=2.44, \mathrm{p}=.022)$; Older children, $0.04(\mathrm{t}(46)=$ $.47, \mathrm{p}=.642) ;$ Adult, $-0.08(\mathrm{t}(23)=1.0, \mathrm{p}=.328)$.

Positive scores for younger children are consistent with Carey's predictions (compare Figure 2 and Figure 5); however, the GENDER x AGE GROUP interaction indicates that the effect of AGE GROUP is only shown by younger girls, not younger boys. For younger girls, AGE GROUP $F(2,50)=5.83, p=.005$; for younger boys, AGE GROUP $F(2,47)=.847, p=.44$.

Summary: The youngest girls show an asymmetry in which they prefer to induce from Human to mammals rather than vice versa. None of the other participants show this asymmetry. Again this may be a familiarity effect: the peccary is a wild animals that young girls who stay by the household are not very familiar with. Young boys who venture into the forest with their fathers are more likely to have seen peccaries on different occasions.

c. Human-Bee asymmetries. The dependent variable for each subject in the ANOVA was their (Human to invertebrate) score minus their (Bee to human) score. The analysis included gender and group as between-subjects factors. There was an effect of AGE GROUP $[F(2,91)=10.97, p<.001]$, but no effect of GENDER. Mean difference score were: Younger children, 0.23 (t-test on $\mathrm{H}->\mathrm{i}$ vs. $\mathrm{B}->\mathrm{h}: \mathrm{t}(25)=2.29, \mathrm{p}=.031)$; Older children, 0.06 $(\mathrm{t}(46)=.68, \mathrm{p}=.50) ;$ Adults, $-0.48(\mathrm{t}(23)=5.47, \mathrm{p}=.0001)$. 
Positive scores indicate that Human to invertebrate is better than Invertebrate to human, which is consistent with Carey's predictions. Posthoc analyses show that adults differ from children, but the children groups do not differ from each other even though the older children show no appreciable asymmetry. Paired samples (within subjects) t-tests show only a difference between Human to invertebrate versus Bee to human inferences for young children.

Summary: There is a developmental shift wherein youngest children show an asymmetry in which they find human-invertebrate inferences (Figure 1) stronger than invertebrate-human inferences (Figure 3). By age 6 this asymmetry has disappeared. By adulthood the asymmetry is reversed so that properties are more readily projected from invertebrates to humans than vice versa. Young children also show a slight asymmetry in favor of projections from Mammals (Dog and Peccary) to invertebrates versus Bee to mammals (Table 1). Although the difference does not reach significance, it may suggest a familiarity effect: human-invertebrate interactions are just more familiar than mammalinvertebrate interactions, and humans and mammals are more familiar than invertebrates as sources of knowledge and inference.

\section{Discussion}

Findings from studies of inductive projection among Yukatek Maya (Figures 1,2,3) do not replicate Carey's results with urban American children (Figures 4, 5, 6) and are not consistent with the claim that folkbiology is decidedly anthropocentric until late childhood. First, for Yukatek Maya children, projections from humans are no stronger than projections from other living kinds. Second, there is no overall asymmetry for Yukatek children as compared to Carey's urban American children. Third, the children do not clearly violate their own perceptions of similarity out of preference for humans as an inductive base. 
There are, however, some asymmetry effects for the youngest girls with respect to a wild versus domestic animal base (Human $->$ mammal $>$ Peccary $->$ human) and for the youngest children overall with respect to inferences involving invertebrates (compare Figures 1 and 4 with Figures 5 and 6). The fact that such asymmetries are not generalized across the youngest age group suggests that they are the result of familiarity effects rather anthropocentric bias as such. Younger girls are less familiar with wild animals than younger boys, and younger children on the whole are less familiar with invertebrates than they are with humans or mammals. Less familiarity with wild animals and invertebrates may favor them less as sources of induction. The fact that dogs are a better base for induction than are peccaries is consistent with this observation.

In important respects the data imply that humans are not a good inductive base for the children. First, the fact that young children (especially the girls) generalize in a fairly undifferentiated way from humans suggests that they may not have a clear grasp of how humans fit into the tree of life. (The young girls show the same pattern with the peccary, an animal with which they are unfamiliar.) Second, older children and adults generalize more from nonhuman mammals to other mammals than they do from humans to mammals. This indicates that humans are, in some respects, special. This is surprising given Carey's findings but consistent with the observation of Johnson, Mervis and Boster (1992) that many children do not think of humans as animals.

A third distinctive pattern that involves humans concerns their special relation to the sun. Younger and older children generalize more from humans to the sun than from any other base to the sun. Adults do not show this pattern. At this stage, it is difficult to distinguish cultural from other developmental factors in interpreting children's projections to the sun. The fact that adult Yukatek accord no inductive preference to sun indicates that the 
developmental pattern is not a simple function of cultural influence. Rather, the sun's culture role in Maya lore may differentially impinge upon the developmental process. A cultural theme like the Maya sun may influence children in ways not seen in the adults of that culture, much as the themes of Santa Clause or God can in our culture (Evans in press, Barrett et al. in press). Until we have comparative data regarding the sun from other cultural populations, however, further speculation would be premature. We will be running additional experiments to fill this void.

On the whole, Yukatek Maya children look much like Menominee children but with some intriguing gender differentiation. These gender differences may reflect the strong sexual division of activity that is institutionalized early in the first year of life: in the jetz' ceremony, Maya girls are introduced by the women to household utensils, whereas Maya boys are introduced by the men to agricultural and hunting tools. Later in life, Maya women will spend their time almost wholly in the vicinity of the house and house garden, in close interaction with domestic animals. By contrast, Maya men spend days, weeks and even months in the forest away from home, and consider animals primarily in terms of their forest habits. For Maya females, dogs are household animals, whereas men value dogs as hunting animals. Maya boys also venture out into the forest with their fathers at an early age, and so become familiar with wild animals, such as the peccary, before girls do.

These latter findings suggest that induction patterns may be influenced by relative familiarity with animals and by the culturally specific character of the functional and ecological relationships between humans and other natural categories of elements. There seems to be additional support for this in the fact that Maya adults more readily project from peccary and bee to humans, and from bee to tree, than do the children. Maya adults consider peccaries and bees to be prime sources of food, and food to be vital to human health and 
physical make-up. Bees primarily make their homes in trees and Maya adults can often tell from a tree's aspect whether bees are likely to be housed in it (and even what honey found inside is likely to taste like). Consistent with this view, Inagaki (1990) presents evidence that knowledge does influence children's biological reasoning. She found that kindergarteners who raised goldfish were more likely than their counterparts who did not raise goldfish to reason about a novel aquatic animal (a frog) by analogy to goldfish rather than by analogy to humans. Thus, knowledge may enable goldfish-raisers to use goldfish as an analogical base in a way that non-goldfish raisers could not.

In sum, even 4-5 Yukatek Maya children show no clear commitment to an anthropocentric understanding of the natural world. Indeed our evidence is more consistent with the view that young children are uncertain about how humans fit into the web of life even while they have a good understanding of how the major life forms are related. The main conceptual change Yukatek children must undergo is not forming an autonomous biology but rather understanding the role of humans on the biology of plants and animals. This shows that folkpsychology is not universally the source for folkbiology. The fact that urban American children do show an anthropocentric bias likely owes more to a difference in cultural exposure to non-human biological kinds than to basic causal understanding of folkbiology per se.

\section{Part II. Inheritance Task}

Young individuals have the potential to develop certain adult characteristics before those characteristics appear. The origins of these characteristics can be explained in two broadly different ways: nature and nurture. Some characteristics seem likely to develop from birth because they are essential to the species to which the individual belongs, such as a squirrel's ability to jump from tree to tree and hide acorns. Other characteristics are 
determined by the environment in which the individual is reared, such as a squirrel's fear or lack of fear of human beings. In this study we examine how children project category-typical as well as unknown behaviors and physical features of animals. We explore whether projections are made on the basis of presumptions of underlying species essence or environmental context, and we examine how presumptions of underlying species essence might sustain the hypothesis that young children have a folkbiology.

Gelman and Wellman (1991) argue that young children predict category-typical characteristics of individual animals based on the innate potential of the animal (i.e. the species of its birth parent) rather than the environment in which it was raised (i.e. the species of its adoptive parent). Using an adoption study, they showed that four-year-old children judge that a baby cow raised by pigs will have the category-typical characteristics of cows (moos, straight tail) rather than pigs (oinks, curly tail). They interpret the results as showing that preschoolers believe that the innate potential or essence of species determines how an individual will develop, even in contrary environments.

This study is inconclusive with regard to children's assumptions about innate potential for two reasons. First, before the children in the study predicted the adult properties of the adopted baby, they were shown a drawing of the baby animal and told its species identity. The children may have projected properties based not on the species of the birth parent, but rather on the basis of the species of the baby itself. Because the experimenters told the child that the baby and mother were of the same species, the study does not address the question of how the children identify to which species the baby belongs in the first place. To demonstrate that the children attribute property origins to inheritance from the birth species requires that the children make the same inferences in the absence of any explicit prior identification of the baby's birth species (see Solomon et al. 1996). Given this explicit 
verbal identification, one cannot rule out that the children's performance owes to an essentialist bias that is a general property of language. Because the animal was labeled as being a member of a particular species, children might expect that this identity is maintained over time, and that the animal would continue to have the properties of the labeled species, without reasoning about the mechanism involved (Gelman \& Hirschfeld 1999). ${ }^{7}$

Second, the study explored only known facts about species and their associated properties. It did not examine whether or not children use the concept of species essence or biological parentage as an inferential framework for interpreting and explaining known and as yet unknown facts. It may be that a child has learned from experience, and as a matter of fact, that a calf is a cow because it was born to a cow. Still, the child may not know that being a member of a certain species causes a cow to be a cow (Carey 1995, Johnson \& Carey 1998). Similarly, a child may learn to correlate other children's toy preferences and gender, and to predict toy preferences on the basis of gender, without necessarily inferring that gender has a causal or explanatory role in toy preferences. In other words, children's performance could be simply explained by their knowledge of input-output relations that are managed by a domain-general learning mechanism.

Johnson and Solomon (1997) designed a set of tasks in part to address the methodological issues associated with Gelman and Wellman's (1991) adoption study. First, 4 to 7-year-old children were told a cross-adoption story accompanied by pictures of each parent. The pictures were sketches that clearly identified the species of each parent, but with minimum detail. Importantly, the experimenter provided no picture of the baby, and did not explicitly identify the baby's species. Second, properties were chosen to minimize factual or pre-learned associations with one or the other species. For example, the child was asked of a baby born to a horse but raised by a cow if it would have a brown nose like the cow or a 
black nose like the horse. Properties included physical traits (e.g., black nose / brown nose), beliefs (did / did not know where special food was kept) and behaviors (e.g., liked to be alone / in crowds). Inclusion of beliefs in addition to physical traits owes its justification to Carey's (1985) studies. Carey's studies suggest that children before the age of ten do not distinguish between the biological and psychological domains in terms of classes of properties (physical traits versus beliefs) that have distinct causal origins. If children do not distinguish between biological and psychological domains, they should not show different patterns of projection of physical and mental properties.

Johnson \& Solomon were interested in whether children would differentiate physical from belief properties by projecting the former from the birth parent and the latter from the adoptive parent. They argue that only the above pattern of property differentiation provides sufficient evidence that children have an inheritance theory of the origin of properties. ${ }^{8}$ The majority of children at all ages failed to show this pattern of property differentiation. The data indicate, however, that across all ages children were more than three times as likely to produce birth bias patterns than adoptive bias patterns (bias patterns are those in which children chose at least 10 of 12 physical, mental, and behavioral properties of the birth or adoptive parent).

In contrast to children, USA adults did show a differentiated pattern - they clearly associated physical properties with the birth parent and mental properties with the adoptive parent. Moreover, for adults behaviors almost always patterned with beliefs. The authors conclude that the children are not able to distinguish classes of animal properties with respect to their domain-specific causal origins. This conclusion is in line with Solomon et al.'s (1996) findings that children do not distinguish the causal origins of physical versus mental properties of people. The results are taken as further support of Carey's $(1985,1995)$ claim 
that there is no early principled (theoretical) distinction between the biological and psychological domains. The authors grant that there may be an essentialist bias to project properties from the birth parent in cases where there are pre-learned associations of kinds with properties, and where children have some factual information about where babies come from (e.g., inside mommies' tummies). Nevertheless, they imply that this essentialist bias does not provide a theoretical basis for inferring causal relationships that involve novel properties. Neither does it have a domain-specific causal origin "because it applies equally well to nonbiological properties such as beliefs" (Johnson \& Solomon 1997:415).

We want to question this line of reasoning. Johnson \& Solomon argue that evidence for an inheritance theory of property origins rests on whether children differentially attribute physical properties and belief properties. However, there are problems with this assumption. First, they assume that children think of animals as having beliefs in the same way that they think of people as having beliefs. This assumption presupposes the identity of the folkbiological and folkpsychological domains; thus the results should not be taken as evidence for that identity. Second, even if children naturally and spontaneously interpret animal behavior in terms of belief-desire psychology, there is no a priori reason to suppose that beliefs should project from the adoptive parent rather than the birth parent. As Gellman and Wellman (1191:216) note: "essential similarities may also be in the form of behaviors, functions, parentage, psychological make-up, or even intangible qualities (e.g., soul)." Third, what is essential and projectible from the birth parent may depend in part on the context and character of the beliefs and behaviors being probed. In the Johnson and Solomon study, behaviors (e.g., did / did not know where special food was kept) and beliefs (e.g., liked to be alone / in crowds) were context-sensitive and sometimes hardly distinguishable (e.g., know versus like both imply propositional attitudes). In the Gelman and Wellman study behaviors 
were context-free (e.g., moos / oinks). Gelman and Wellman actually found behaviors to be more reliable predictors of species kind than physical features.

We argue that sufficient evidence that a specifically biological framework underlies causal assumptions about property origins does not require attribution of beliefs to adoptive parents. Instead, we hold that a biological interpretation of property origins requires that children assume that certain kinds of properties are attributed to birth parents rather than adoptive parents despite the lack of prior association between the property and the category. Our cross-cultural study was designed to test the extent to which children's assumptions about innate species potential govern projection of both known and unknown properties. The current study was designed to avoid the problems noted for these earlier studies in testing the hypothesis that young children have an essentialist understanding of biological phenomena and, therefore, that they already have a causally conceptualized folkbiology.

\section{Method}

\section{Participants}

Participants were 48 Yukatek Maya-speaking children and 24 Yukatek Mayaspeaking adults $(M=33$ years; range $=26$ to 40$)$. Twenty-four 4 -to-5 year olds $(M=5 ; 3$, range $=4 ; 6$ to $5 ; 11)$ and twenty-four 6 -to- 7 year olds $(M=7 ; 1$, range $=6 ; 7$ to $7 ; 7)$ were tested and included. An equal number of males and females was included in each group. By and large, the 4-5 year-olds were monolingual, the 6-7 year-olds had begun learning Spanish, and alnmost all of the adults understood Spanish as a second language. All testing was done in Yukatek Maya.

\section{Procedure}

In a forced choice task, children were asked whether an adult animal adopted at birth would resemble its adoptive parent (e.g., cow) or birth parent (e.g., pig) on four different 
individual traits (see Appendix A for the text of the story): known behaviors (e.g. moo / oink), known physical features (e.g. straight / curly tail), unknown, arbitrary behaviors (e.g. looks for cardinals / looks for sparrows), and unknown physical features (e.g. heart gets flatter / rounder when it is sleeping). Known traits were context-free, category-typical features that the children readily associated with species, whereas unknown traits were chosen to minimize any possibility of factual or pre-learned associations of traits with categories. Each unknown trait within a set was attributed to the birth parent for half the participants and to the adoptive parent for the other half. This assured that projection patterns of the unknown traits were not based on prior associations.

The stories were accompanied by sketches of each parent (see Figure 7). As in the Johnson and Solomon study, sketches were designed to unambiguously represent a particular species of animal with minimum detail. In addition, as shown in Figures 8 and 9, sketches of known physical features (e.g. a sketch of a curly or straight tail), unknown physical features (e.g. flat vs. round heart) and relevant aspects of unknown behavioral contexts (e.g., closed vs. open eyes, mahogany vs. cedar tree) were also shown to the participant. These sketches in no way indicated the species to which the traits belonged. Sketches of traits were placed beside the sketches of the species they were being attributed to. Participants indicated their choice of birth or adoptive parent species by pointing to the relevant parent sketch.

The story was followed by two comprehension controls: a birth control (Who gave birth to the baby? Go ahead and point out the drawing of who gave birth to the baby.) and a nurture control (Who did the baby grow up with?) If the child failed either control the adoption story was repeated and a second failure in comprehension resulted in exclusion of the child from the experiment. Children then were presented with the four experimental probes (see Appendix B). For example: The cow mooed and the pig oinked. When the baby 
is all grown up will it moo like a cow or oink like a pig? For each set, the four probes were followed by a bias control in which the participant was asked: When the baby was growing up did it eat with animals that looked like X or animals that looked like Y? Go ahead and point out the animal that looks like the animals the baby grew up eating with. Notice that the bias control is not simply a memory check, but requires the child to generate an inference about the relationship between nurturing, in general, and eating, in particular.

The same procedure was used with all three sets. The order of the four trait probes was randomized across participants, as was the order of presentation of the three sets. The order of presentation of parents within each probe was held constant for each child and counterbalanced across children.

Following presentation of the three sets on the four trait probes and the bias control children were asked: "Now the baby is all grown up, what kind of animal is it? Go ahead and point out the kind of animal the baby grew up to be." The additional probe for kindhood was designed to test Johnson and Solomon finding that 4-year-olds reliably attributed kindhood to the birth parent. In Yukatek Maya there is no direct gloss for "kind." Instead, the Mayanized Spanish word klaasej was used.

A final probe involved a transformation story to explore the extent to which species essences are associated with inheritance versus vital internal properties as such (i.e., blood, see Appendix A). Keil (1989:224), found that the younger children are undecided as to whether inheritance or internal properties are primarily responsible for an animal's species identity.

\section{Results}

For each probe, participants were given a score of one if they chose the birth parent and zero if they chose the adoptive parent. A 2 (gender) x 3 (age group) x 3 (set) x 6 (probe 
type: known behavior, known physical feature, unknown behavior, unknown physical feature, blood, and kind) repeated measures ANOVA indicated only a main effect of probe type, $F(5,62)=3.9, \mathrm{p}<.01$. Probe type means are shown in Table 2 . Each mean was tested against chance (0.5) and the results are indicated in Table 2.

Overall, the results show a systematic and robust preference for attributions from the birth parent. This preference was observed for all age groups and for known and unknown behavior and physical properties. The trend is somewhat stronger in older children and adults and slightly stronger for known than unknown properties. Means for all probes were significantly different from chance, except the kind and blood for the youngest children. The kind probe was only marginally different from chance for the young children $(p=0.10)$, possibly because of foreign character of the Mayanized Spanish word for kind, klaasej. Results on the blood probe for the youngest children might suggest genuine indecision as to whether inheritance or vital internal functioning is primarily responsible for an animal's species identity. The low mean on the bias control probe for all groups indicates that the method of the current experiment did not bias participant responses toward the birth parent.

To measure the effect of the different trait probes, a 3 (AGE GROUP) x 2 (GENDER) x 3 (SET) x 2 (known vs. unknown trait) x 2 (behavioral vs. physical trait) repeated measures ANOVA showed only a main effect of the familiarity of the probe (known $=0.88$ and unknown $=0.81)[\mathrm{F}(1,66)=9.5, \mathrm{p}=0.003]$. Across groups, children and adults are more likely to attribute known than unknown properties to the birth parent.

In order to measure whether there was a difference in the strength of the birth parent bias across groups, percent birth parent attributions was calculated for each participant across trait types (all probes except the bias control probe). A one-way ANOVA with AGE GROUP as the independent factor and BIRTH BIAS as the dependent variable showed an effect of 
group $[F(2,71)=23.3, p<.001]$. Tukey post hoc tests showed that the strength of the BIRTH BIAS was stronger for older children (0.89) and adults (0.94), who did not differ from one another, than for younger children (0.67).

In conclusion, the results of this study suggest that Yukatek Maya children and adults assume that members of a species share an innate causal potential that largely determines category-typical behavioral and physical properties even in conflicting environments. The current study is a first demonstration that members of another culture systematically use this notion of species essence to project unknown properties in the face of uncertainty. Projection of properties to the birth parent in the face of uncertainty and novelty implies that even young Maya children use the notion of underlying essence as an inferential framework for understanding the nature of biological species. ${ }^{9}$ By the age of seven, children have effectively attained adult competence in inferential use of the notion of innate species potential.

\section{Discussion}

One of the more difficult issues in theoretical disputes is separating conflicts over matters of fact from conflicts over meaning. The dispute here is over the criterion of demarcation for what can be considered a folkbiology. For Carey, demarcation involves the notion of intuitive framework theory. The attribution of an intuitive framework theory to young child "requires establishing that the child distinguishes entities in the domain of the theory from those not in its domain, and appeals to theory-specific causal mechanisms to explain the interactions among the entities in the domain" (Carey 1995). On this view, attributing a folkbiology to young children entails attributing a biological causal mechanism that delimits an ontological domain.

So far, we agree. But Carey's account also implicitly involves two a priori claims 
with which we do not agree: namely, that (1) causal understanding does not exist in the absence of any detailed knowledge of specific causal laws or mechanisms, and (2) essentialism is too causally vague and domain-general to distinguish biology. Concerning the first claim, the minimum conditions that Carey sets for a properly biological notion of causality tend to overplay the causal mechanisms that adults use to understand biological phenomena: "pre-school children have learned that 'germs' are a cause of disease, but we do not know whether this knowledge goes beyond naming 'germs' as the cause of disease.... Such knowledge may simply be a learned input-output relation, such as that eating good foods keeps you healthy and makes you grow, and may not constitute knowledge of any mechanism" (Carey 1995:284; cf. Kalish 1993). Thus, 'germ' cannot count as part of a causal mechanism because there is no understanding of the specific processes involved. Ordinary adults, however, may have a barely more elaborate causal understanding of germs or genetics. ${ }^{10}$ The problem here is not simply that of overestimating adult knowledge, but of implicitly subordinating the notion of causal mechanism to a preconceived standard of explicit detail.

Concerning the second claim, Carey holds that essentialism is not a serious candidate for causally organizing the biological domain because it is simply a general property of language: "Essentialism, like taxonomic structure, derives from the logical work done by nouns. The child has a default assumption that... every count noun carries with it the idea that the identity of the entity picked out by the noun is unchanged in the face of surface changes" (Carey 1995:277). By 18 months of age, for example, children are able to consistently apply nouns to persons, animals and substances identified over time (Macnamara 1982, 1986). Nevertheless, this is a different sense of essentialism than the concept of innate causal and inductive potential that we (and others) intend. 
Our use of essentialism to describe the causal underpinnings of folkbiology conforms to the idea of "psychological essentialism” formulated by Medin and Ortony (1989).

Psychological essentialism is a framework theory that defines the ontology of a domain and places limits on the kinds of information and causal mechanisms that specific theories can incorporate. The central claim of psychological essentialism is that surface features may be seen as effects caused by deeper, underlying features even in the absence of knowledge about specific causal mechanisms. Biological essentialism is a particular version of psychological essentialism: “The essential trait, or nature, (e.g., the peculiar felinity of tigers) 'underlies' the better known perceptible features (e.g., being large and striped); that is, the perceptual features of a kind are presumed to be natural consequences of, or to be naturally caused by, the essential nature of that kind, even if the essential nature is largely unknown and perhaps effectively unknowable" (Atran 1987:44). In this version of essentialism, there might not even be a set of defining features at the surface level because essences might not always generate necessary and sufficient properties. Something could be part of an essence but not necessarily manifest the associated surface properties. For example, tigers could be essentially described as large, striped quadrupeds that roar, but circumstances might conspire such that a given tiger develops as a mute, as three-legged, as an albino, or as a dwarf. A tiger may still be theoretically and virtually quadrupedal (i.e., in virtue of its underlying nature) even if only three-legged in actual appearance.

By contrast, Carey's (1995) notion of linguistic essentialism does not require a “deepcause" structure. On her definition of essentialism, all that is required is some sort of maintenance of identity over time. This notion of essentialism does not distinguish between presumptively complex causal concepts, such as "oak" or "robin", and presumptively simple causal concepts, such as "seat" or "hill" that depend only on surface features that are 
practically identical with underlying "essence": a seat is a seat because it can be sat upon, no matter whoever or whatever made it, and no matter whatever it is made of; a hill is a hill because it is higher than the surrounding landscape but lower than a mountain, whatever different and independent causes might be responsible for such a state of affairs. ${ }^{11}$ Because this relatively unconstrained definition of essentialism extends to nonbiological concepts, it follows that this notion of essentialism cannot be the demarcating criterion for folkbiology. This is a matter of semantics, not fact. ${ }^{12}$

Our hypothesis is that from a quite early age children have intuitions that the mechanisms underlying essential causes are biological. The essential causal relations are those involving, for example, birth, biological relatedness and internal structure. It is unlikely that young children have a worked out a specific theory or detailed model that integrates inheritance, growth, physiological functioning, disease, death and so forth ; however, it may be plausible to credit them with a generally biological framework. The details likely change with development and vary cross-culturally. Thus, it may well be that people in all cultures assign every individual nonhuman living kind to one and only one folk species (also called "generic" or "generic species") on the basis of a universal causal presumption that local biodiversity is divided into essential kinds (Atran 1998). But people in different cultures may attribute very different contents and causes to species essences. For example, Itza' Maya Indians, whose everyday lives depend upon knowledge of biological kinds, appear to have much richer knowledge and causal inferences associated with folk species than do urban or rural Americans (Lopez, et al. 1997, Coley et al. 1997, Atran et al. 1997). Nevertheless, Itza' Maya and Americans readily agree on the common folk species present in their respective environments, and also agree that biological information and inference is best had at the folkspecies level (e.g., at the level of dog and sparrow as opposed to subordinate level of 
doberman and song sparrow or the superordinate level of mammal and tree). Similarly, Japanese and American children acknowledge many of the same species, although Japanese children appear to have much more elaborated vitalist theories of what causes, for instance, a given dog to be a dog (Hatano \& Inagaki 1999).

\section{Conclusion}

It is a troubling fact that nearly all psychological research on basic cognitive processes of category formation and reasoning is based on work with populations associated with large research institutions in technologically-advanced societies. It would be hard to come up with a more culturally-restricted subject pool from which to generalize to humanity as a whole. Lopsided attention to a select participant pool risks biasing interpretation, no matter how large the sample population or how statistically reliable the results.

With regard to biological understanding, the effect of this bias may be aggravated by people's relative lack of direct contact with nature's species. Much of what folk in our societies know about animals, for example, comes from books and television programs that focus on domestic animals and non-native mammals. This is a poor and fragmentary sample compared to the natural conditions under which humanity's cognitive capacities evolved and most human cultures developed and diversified. The pitfalls of generalizing about biological knowledge from this one cultural sample - however large or convenient- might be comparable to the perils of generalizing about human language just from studies of feral children.

Nevertheless, such generalization is routine and rarely questioned in the major journals that serve as research outlets. Doing otherwise would present serious inconveniences. Instead of handing out questionnaires to undergraduate psychology students or sending a research assistant down the street to a local school, one would have to learn the 
languages, habits, preferences and biases of perfect strangers. This is a daunting and expensive enterprise to be sure. Unfortunately, good science sometimes requires costly efforts.

Whether or not the experiments reported here represent good science is for the reader to judge. We have at least tried to show that the effort is worthwhile, by reporting findings that seriously call into question the empirical generality and theoretical importance of conclusions based exclusively or primarily on studies of people from our own society and the cultural milieu most accessible to us. Of course, we are not the only ones to make the effort. Psychologists Susan Carey and Greg Solomon have teamed up with anthropologists Maurice Bloch and Rita Astuti in an ambitious cross-cultural research project that promises new insights into the subtle but pervasive effects of cultural experience on acquisition of biological knowledge. As their results come in, the debate on the origins and development of biological knowledge will likely reach a new level of sophistication and perhaps an unexpected resolution. Other cross-cultural and cross-disciplinary research efforts are now underway. The new Journal of Cognition and Culture comes as a timely carrier and spur for us all. 
NOTES 
Table 1: Means for Yukatek Maya Induction Task

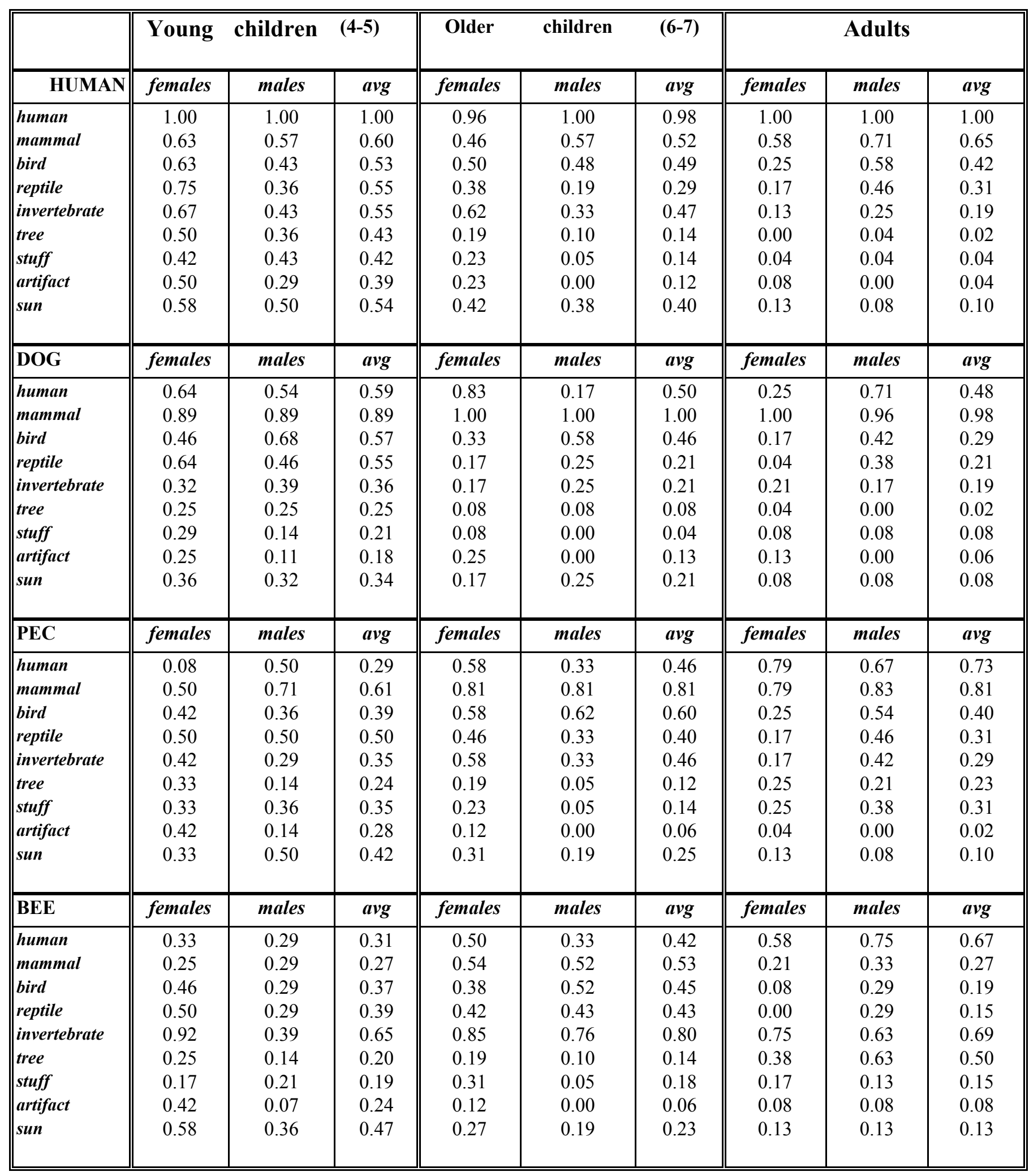


Table 2. Percent birth parent choice for each probe type for each group

\begin{tabular}{|c|c|c|c|c|c|c|c|c|c|}
\hline \multirow[b]{2}{*}{ GROUP } & \multicolumn{3}{|c|}{ Known } & \multicolumn{3}{|c|}{ Unknown } & \multirow[b]{2}{*}{ KIND } & \multirow[b]{2}{*}{ BLOOD } & \multirow[b]{2}{*}{$\begin{array}{l}\text { Bias Control } \\
\text { (Food) }\end{array}$} \\
\hline & behavior & phys feat & mean & behavior & phys feat & mean & & & \\
\hline $4-5$ year olds & $0.74 * *$ & $0.68^{*}$ & 0.71 & $0.69 * *$ & $0.68^{*}$ & 0.69 & 0.65 & 0.56 & $0.06^{* * *}$ \\
\hline 6-7 year olds & $0.96^{* * *}$ & $0.97 * * *$ & 0.97 & $0.82 * * *$ & $0.83 * * *$ & 0.83 & $0.99 * * *$ & $0.79 * *$ & $0.01 * * *$ \\
\hline adults & $1.0 * * *$ & $0.96 * * *$ & 0.98 & $0.90 * * *$ & $0.93 * * *$ & 0.92 & $0.97 * * *$ & $0.88 * * *$ & $0 * * *$ \\
\hline Mean & 0.90 & 0.87 & 0.88 & 0.81 & 0.81 & 0.81 & 0.87 & 0.74 & 0.02 \\
\hline
\end{tabular}

$\mathrm{p}<0.05^{*}, \mathrm{p}<0.01^{* *}, \mathrm{p}<0.001^{* * *}$ 


\section{Appendix A}

\section{WARM-UP QUESTION}

A baby deer grew up with other deer in the forest. When the baby is all grown up will it to drink water or coffee? Will it be brown or green?

\section{ADOPTION STORY}

\{Paired Items X / Y: COW / PIG, PIGEON / TURKEY, TURTLE / TOAD\}

I'm going to tell you a story. One day a X (e.g., cow) gave birth to a little baby. Here's a drawing of the X (e.g., the cow) that gave birth to the baby [child sees drawing of $\mathrm{X}$ ]. Right after the baby was born the X (e.g., the cow) died without ever seeing the baby [drawing of $\mathrm{X}$ is removed].

The baby was found and taken right away to live with Ys (e.g., pigs) in a place where there are lots of Ys (e.g., pigs). Here's a drawing of the Y (e.g., the pig) [child sees drawing of $\mathrm{Y}$ ] which took care of the baby the whole time that the baby was growing up [drawing of $\mathrm{Y}$ is removed]. The baby grew up with Ys (e.g., pigs) and never saw another X (e.g., cow) again.

Now the baby is all grown up and I'm going to ask some questions about what it's like as an adult.

\section{FOLLOW-UP QUESTION ABOUT THE RELATION OF SPECIES KIND TO INTERNAL FUNCTION-STRUCTURE}

When the baby was growing up it became sick. A doctor came and, with a needle, took out all of the old blood that the baby got from its mother [show drawing of birth parent, $\mathrm{X}$ ] when it was born. The doctor then went to the animal that was taking care of the baby [show drawing of adoptive parent $\mathrm{Y}]$ and took some of its blood to give to the baby. So the baby got all new blood like the blood of $\mathrm{Y}$.

Now the baby is all grown up, what kind of animal is it? Go ahead and point out the kind of animal the baby grew up to be. 


\section{Appendix B}

Known Traits

TURTLE

Phys feature shell on its back

Behavior walks slowly

PIGEON

Phys feature short neck

Behavior very used to flying high COW

Phys feature straight tail

Behavior moo

\section{Unknown Traits}

\section{TURTLE / TOAD}

Phys feature stomach gets harder when it sleeps

Behavior opens its eyes when afraid

\section{PIGEON / TURKEY}

Phys feature blood becomes thick and sticky when it sleeps

Behavior stops when it sees a mahogany tree

\section{COW / PIG}

Phys feature heart gets flatter when it sleeps

Behavior

\section{TOAD}

warts on its back

hops

\section{TURKEY}

long neck

very used to running on ground

PIG

curly tail

oink

stomach gets softer when it sleeps

closes its eyes when afraid

blood becomes thin and watery when it sleeps

stops when it sees a cedar tree

heart gets rounder when it sleeps

looks for pigeons 
Figure 7

\section{Examples of Pictures of Mother Animals}
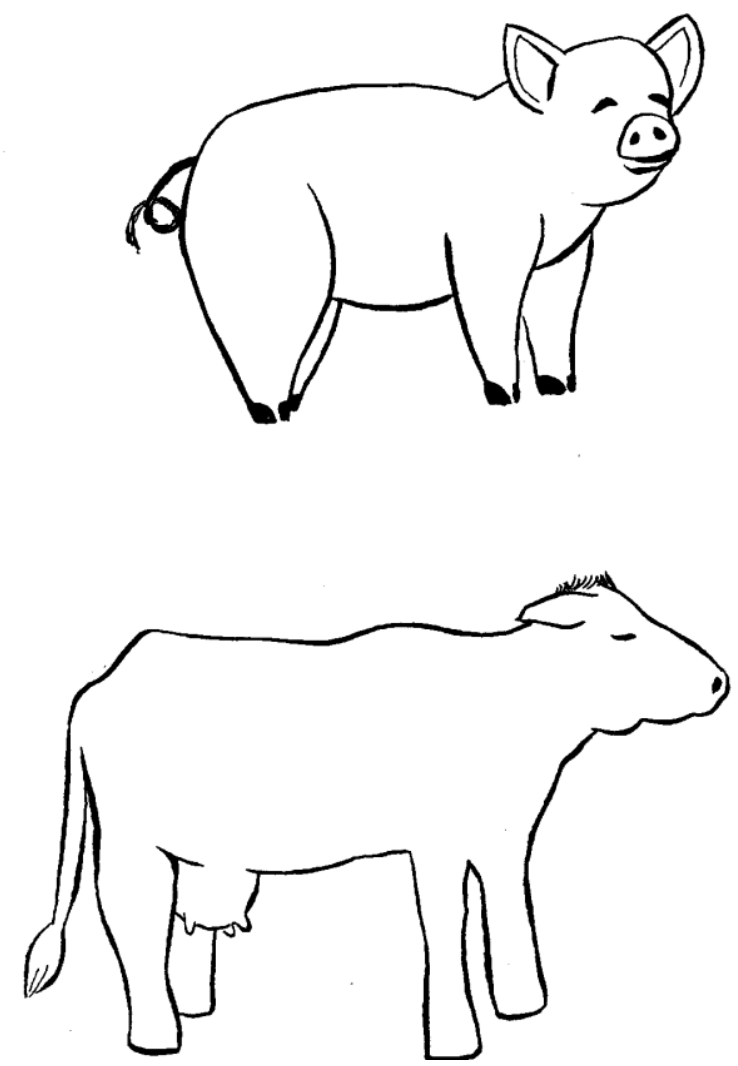


\section{Figure 8}

Examples of Known Traits (straight vs. curly tail)
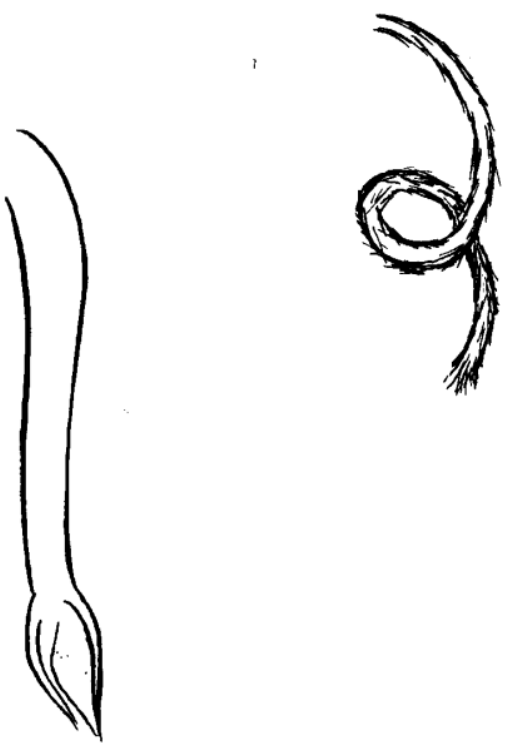


\section{Figure 9}

\section{Examples of Unknown Traits (flat vs. round heart)}
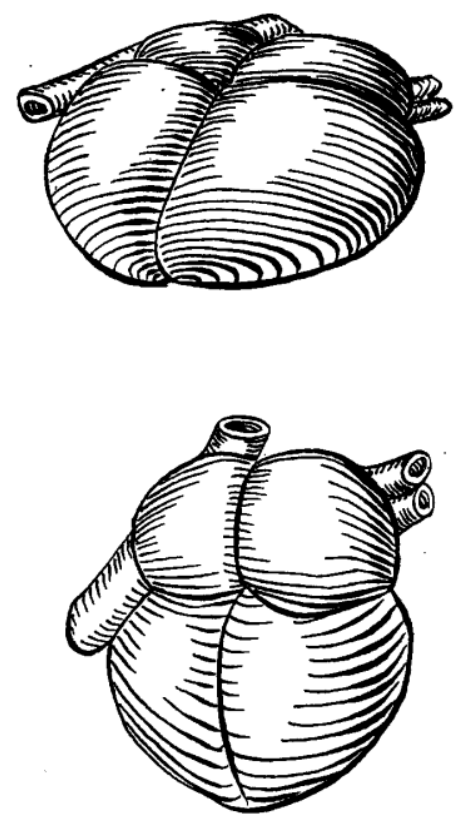

${ }^{1}$ This research was supported in part by NSF grant SBR-9707761 to S.A. and D.M. and time provided by the Leverhulme Visiting Professorship at the University of London-Goldsmiths to S.A. We wish to thank Susan Carey, Susan Gelman, Lawrence Hirschfeld, Greg Solomon and Dan Sperber for comments on earlier drafts. Email for correspondence:

satran@umich.edu.

${ }^{2}$ Carey (1995) acknowledges that biology may depart from psychology even earlier than age 7, and intimates that biology and psychology may initially constitute a single undifferentiated 
domain of causal understanding. The implications of this view are not clear. No new predictions follow about the causal structure and ontological composition of either domain that are not present in Carey's (1985) earlier account (except for the ad hoc prediction that the two domains diverge at an earlier age).

${ }^{3}$ More extended versions of the independence of biological causality apply to humans as well as nonhumans (Hirschfeld 1994, 1996; Springer 1992, 1996; cf. Weissman \& Kalish 1999). In these versions, children also differentiate human biology from human psychology early on. It is not at all apparent, however, that the putative biological structures of individual humans, kin groups, and nonkin social groups (e.g., races) are cognitively processed in the same way as the biological organization of nonhuman species and their members. For example, nonhuman individuals appear to be universally and "automatically" processed as exemplars of the unique species they instantiate. By contrast, individual humans are not primarily or invariably processed as biological exemplars of one and only one group, much less as exemplars of taxonomic equivalence classes that fully and uniquely partition the human domain (Atran 1998). In any event, the experiments reported in this article do not necessarily rule out, or generalize to, nontaxonomic and extended versions of folkbiology that apply also to humans.

${ }^{4}$ For those organisms that are phenomenally salient for human beings, such as most vertebrates and flowering plants, there is usually only one representative species of a biological genus in any given locale. Because biological genus and species are often extensionally equivalent in a locality, there is no conceptual basis for a consistent folkbiological distinction between them. Such a distinction emerged only during Europe's Age of Exploration, when foreign species were integrated into the taxonomic system by 
attaching them to a European type-species at the genus level (Atran 1990).

${ }^{5}$ Such universal aspects of folkbiology may represent evolutionary constraints on any and all theorizing in biology, including evolutionary theory itself (Atran 1998). If the end state of acquired knowledge is everywhere structurally similar in design, and functionally critical to dealings with multiple environments, then it is plausibly an evolutionary adaptation to relevant and recurrent aspects of ancestral hominid conditions - such as the need of farranging hunters and foragers to recognize, avoid and exploit indefinitely many ambient species.

${ }^{6}$ This methodology applied to a sample of urban Boston children replicated Carey's findings in important respects. Carey found significant differences between strength of projections from humans to categories in the same class (e.g., aardvark, dog) as the base (e.g., dog), and the strength of projection from the non-human base (dog) to humans for 4-year-olds (e.g., average difference Human $>$ mammal minus Mammal $>$ human $=50 \%$ ) and 6-year-olds (20\%). Coley et al. also find an asymmetry for Boston 4-year-olds (26\%) and 6-year-olds $(39 \%)$.

${ }^{7}$ In another study, however, Gelman and Wellman (1991) asked children to reason about plants without identifying the species membership. For example, they described a seed that came from an apple and was planted in a field a corn, without identifying the seed as "an apple seed." The results were largely the same as with the animals (cf. Hickling \& Gelman 1995).

${ }^{8}$ Earlier work by Solomon et al. (1996) that established the property-differentiation paradigm was criticized by Springer (1996) as having an overly complex methodology. When Springer reduced the task demands in the switched-at-birth design by simplifying the adoption story, a 
fairly robust nativist position emerged. In a task where the story was modified to exclude explicit reference to adoption, preschoolers showed a greater tendency to attribute physical properties to birth parents than non-physical properties (e.g., beliefs); however, there was no overall birth bias even for physical properties (see also Hirschfeld 1994). The relevance of these studies for our experiments are not clear inasmuch as all the stories used were heavily anthropomorphic and involved many implicit but critically unexamined assumptions about animals having preferences, beliefs, propositional attitudes and so on. In any event, Johnson and Solomon's (1997) study avoids some of the earlier problems noted by Springer. For example, their story does not mention "adoption" and is structured in ways quite similar to Springer's modified story.

${ }^{9}$ This inheritance study was performed with three different groups of 4-5 year-olds in Brazil. Preliminary analysis shows a pattern of results very similar to Yukatek Maya 4-5 year-olds. ${ }^{10}$ This example illustrates another problem with Carey's idea of an implicit theory or explanatory framework. The claim that the knowledge that germs causes diseases is only a knowledge of input-output relations blurs the distinction between theory and tabulation of observable regularities that is fundamental to Carey's approach. Germs are not observable entities in this context and ought to be classified as theoretical entities. Granted there is no clear or neat distinction between observable entities and non-observable entities, or between empirical laws and theories; however, without this distinction in principle, the notion of intuitive theory becomes incoherent.

11 Carey's linguistic essentialism conforms to certain aspects of Streven's (2000) account of “minimalist" essentialism. Psychological essentialism and minimalist essentialism both allow that children may have little, if any, idea of specific causal mechanisms. According to 
Strevens (2000:163): "This is not to say that children think that there are no essences; rather, they have no opinion about what it is that makes the causal laws true." This suggestion resemble's Medin and Ortony's (1989) idea of an “ essence placeholder”; however, there is a difference. On Strevens' minimalist account, no concept of "common-cause" is needed to explain children's performance. Strevens' essentialism is even more liberal than Carey's in that mass terms, such as "mud" and "red things," may be just as good candidates for essentialism as count terms. Thus, red things share the disjunctive "essence" of whatever causes them to be red: red stars are red because of the way light filters through the earth's atmosphere to our retinas; British telephone booths are red because they are painted red; male sticklebacks are red in part because of their DNA, and so on. Muddy things share the conjunctive "essence of being soft, wet, slimy, sticky earth, although several different and independent causes may be involved. All that is needed is the presumption that something causes surface features. That something may have divided reference: one thing can cause a lion to roar and another thing, unrelated to the first, can cause a lion to have a mane. Furthermore, any given surface feature might have more than one cause: two or more different things might cause a lion to roar. Finally, some causes might be deep and others superficial, such as believing a male lion's mane is genetically caused versus believing that it produced by fright, grooming by female lions or other external agents. Neither Carey nor Strevens provide clear descriptions of what they mean by an essence, except to allow essences to comprehend concepts that do not depend upon deep or common-cause. For a modal account of biological essentialism, see Atran 1987.

${ }^{12}$ Gelman and Hirschfeld (1999) propose a classification of types of essentialism, specifically distinguishing between sortal essentialism (the "classical" view of concepts as 
defined by necessary and sufficient conditions) and causal essentialism (the unknown and perhaps unknowable principles that are physically responsible for something being what it

is). This distinction does not capture the nuances between the various types of causal relationships pertinent to our discussion of the differences between Carey, Strevens, and

\section{Medin and Ortony. REFERENCES}

Atran, S. (1987) Ordinary constraints on the semantics of living kinds: A commonsense alternative to recent treatments of natural-object terms. Mind and Language 2:27-63.

Atran, S. (1990) Cognitive foundations of natural history: Towards an anthropology of science. Cambridge, England: Cambridge University Press.

Atran, S. (1998) Folkbiology and the anthropology of science. Behavioral and Brain Sciences 21:547-609.

Atran, S. (1999) Itzaj Maya folk-biological taxonomy. In D. Medin \& S. Atran (eds.), Folk biology. Cambridge MA: MIT Press.

Atran, S., Estin, P., Coley, J. \& Medin, D. (1997) Generic species and basic levels: Essence and appearance in folk biology. Journal of Ethnobiology 17:22-45.

Au, T. \& Romo, L. (1999) Mechanical causality in children's « folkbiology. » In D. Medin \& S. Atran (eds.) Folbiology. Cambridge, MA: MIT Press.

Baron-Cohen, S. (1995) Mindblindness: An essay on autism and theory of mind. Cambridge, MA: MIT Press.

Barrett, J., Richert, R. \& Driesenga, A. (in press) God's beliefs versus mother's: The development of non-human agent concepts. Child Development.

Berlin, B. (1992) Ethnobiological classification. Princeton: Princeton University.

Berlin, B., Breedlove, D., \& Raven, P. (1973) General principles of classification and nomenclature in folk biology. American Anthropologist 74:214-242.

Brown, C. (1984) Language and living things: Uniformities in folk classification and naming. New 
Brunswick NJ: Rutgers University Press.

Carey, S. (1985) Conceptual change in childhood. Cambridge MA: MIT Press.

Carey, S. (1995) On the origins of causal understanding. In D. Sperber, D. Premack \& A. Premack (eds.), Causal cognition. Oxford: Oxford University Press.

Carey, S., \& Spelke, E. (1994). Domain-specific knowledge and conceptual change. In L. Hirschfeld \& S.Gelman (eds.), Mapping the mind: Domain specificity in cognition and culture New York: Cambridge University Press.

Coley, J., Medin, D. \& Atran, S. (1997) Does rank have its privilege? Inductive inferences in

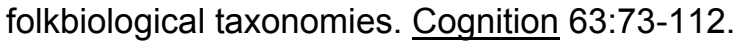

Coley, J., Medin, D. \& James, L. (1999) Folkbiological induction among Native American children. Paper presented at the Biennial Meeting of the Society for Research in Child Development, Albuquerque, NM.

Darwin, C. (1883) On the origins of species by means of natural selection, 6th ed. New York: Appleton (originally published 1872)

Dougherty, J. (1979) Learning names for plants and plants for names. Anthropological Linguistics 21:298-315.

Evans, M. (in press) The emergence of diverse belief systems: Creation versus evolution.

Cognitive Psychology.

Gelman, S. \& Hirschfeld, L. (1999) How biological is essentialism? In D. Medin \& S. Atran (eds.) Folbiology. Cambridge, MA: MIT Press.

Gelman, S. \& Wellman, H. (1991) Insides and essences. Cognition 38:214-244.

Gopnik, A. \& Wellman, H. (1994) The theory theory. In L. Hirschfeld \& S.Gelman (eds.), Mapping the mind: Domain specificity in cognition and culture New York: Cambridge University Press.

Hatano, G. \& Inagaki, K. (1994) Young children's naive theory of biology. Cognition 50:171-188.

Hatano, G. \& Inagaki, K. (1999) A developmental perspective on informal biology. In D. Medin \& S. Atran (Eds.), Folk biology. Cambridge MA: MIT Press. 
Hays, T. (1983) Ndumba folkbiology and general principles of ethnobotanical classification and nomenclature. American Anthropologist 85:592-611.

Hickling, A. \& Gelman, S. (1995) How does your garden grow? Evidence of an early conception of

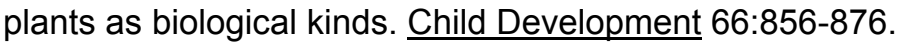

Hirschfeld, L. (1994) The child's representation of human groups. In D. Medin (ed.), The psychology of learning and motivation: Advances in research and theory, vol. 30. New York: Academic Press;

Hirschfeld, L. (1996) Race in the making. Cambridge, MA: MIT Press.

Hunn, E. (1977) Tzeltal folk zoology. New York: Academic Press.

Inagaki, K. (1990) The effects of raising animals on children's biological knowledge. British Journal of Developmental Psychology 8:119-129.

Johnson, K., Mervis, C. \& Boster, J. (1992). Developmental changes within the structure of the mammal domain. Developmental Psychology 28:74-83.

Johnson, S. \& Carey, S. (1998) Knowledge enrichment and conceptual change in folkbiology: Evidence from people with Williams Syndrome. Cognitive Psychology 37:156-200.

Johnson, S. \& Solomon, G. (1997) Why dogs have puppies and cats have kittens: The role of birth in young children's understanding of biological origins. Child Development 68:404-419.

Kalish, C. (1993) Preschoolers understanding of germs as causes of illness. Paper presented at the Biennial Meeting of the Society for Research in Child Development, New Orleans, LA.

Keil, F. (1989) Concepts, kinds, and cognitive development. Cambridge MA: MIT Press.

Keil, F. (1995) The growth of causal understandings of natural kinds. In D. Sperber, D. Premack \& A. Premack (eds.), Causal Cognition. Oxford: Clarendon.

Leslie, A. (1994) ToMM, ToBy, and agency: Core architecture and domain specificity. In L. Hirschfeld \& S. Gelman (eds.) Mapping the mind: Domain-specificity in cognition and culture. New York: Cambridge University Press.

López, A., Atran, S., Coley, J., Medin, D., \& Smith, E. (1997) The tree of life: Universals of folk- 
biological taxonomies and inductions. Cognitive Psychology 32:251-295.

Macnamara, J. (1982) Names for things: A study of human learning. Cambridge, MA: MIT Press. Macnamara, J. (1986) Border dispute: The place of logic in psychology. Cambridge, MA: MIT Press.

Medin, D., Lynch, E., Coley, J. \& Atran, S. (1997) Categorization and reasoning among tree experts: Do all roads lead to Rome? Cognitive Psychology 32:49-96.

Medin, D. L., \& Ortony, A. (1989). Psychological essentialism. In S. Vosniadou and A. Ortony (eds.), Similarity and analogical reasoning. New York: Cambridge University Press.

Proffitt, J., Coley, J. \& Medin, D. (2000) Expertise and category-based induction. Journal of Experimental Psychology: Learning, Memory, and Cognition 25:811-828.

Rosch, E., Mervis, C., Grey, W., Johnson, D., \& Boyes-Braem, P. (1976) Basic objects in natural categories. Cognitive Psychology 8:382-439.

Solomon, G., Johnson, S., Zaitchik, D. \& Carey, S. (1996) Like father, like son: Young children's understanding of how and why offspring resemble their parents. Child Development 67:151-171.

Springer, K. (1992) Children's beliefs about the biological implications of kinship. $\underline{\text { Child }}$ Development 63:950-959.

Springer, K. (1996) Young children's understanding of a biological basis for parent-offspring relations. Child Development 67:2841-2856.

Strevens, M. (2000) The naïve aspect of essentialist theories. Cognition 74:149-175.

Stross, B. (1973) Acquisition of botanical terminology by Tzeltal children. In M. Edmonson (Ed.) Meaning in Mayan languages. The Hague: Mouton.

Weissman, M. \& Kalish, C. (1999). The inheritance of desired characteristics: Children's view of the role of intention in parent-offspring resemblance. Journal of Experimental Child Psychology 73:245-265.

Wimmer, H. \& Perner, J. (1983) Beliefs about beliefs: Representation and constraining function of wrong beliefs in children's understanding of deception. Cognition 13:103-128. 
